\section{ATU-08 GENETIC STUDIES OF MRI LIVER FAT CONTENT IDENTIFY SUSCEPTIBILITY VARIANTS WITH VARIABLE METABOLIC EFFECTS}

${ }^{1}$ Constantinos Parisinos ${ }^{*},{ }^{2,3}$ Henry Wilman, ${ }^{3}$ Matt Kelly, ${ }^{5}$ Stefan Neubauer, ${ }^{2}$ Louise Thomas, ${ }^{2}$ Jimmy Bell, ${ }^{1}$ Aroon Hingorani, ${ }^{1}$ Riyaz Patel, ${ }^{1}$ Harry Hemingway, ${ }^{3}$ Rajarshi Banerjee,

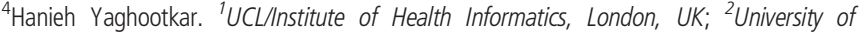
Westminster, London, UK; ${ }^{3}$ Perspectum Diagnostics, Oxford, UK; ${ }^{4}$ University of Exeter, Exeter, UK; ${ }^{5}$ University of Oxford, Oxford, UK

\subsection{6/gutjnl-2019-BSGAbstracts.208}

Background and aims Excess liver fat affects up to 1 in 4 adults globally ${ }^{1}$ and has been implicated in the pathogenesis of liver disease including cirrhosis and hepatocellular carcinoma, as well as extrahepatic diseases such as type 2 diabetes and cardiovascular diseases ${ }^{2}$. We aimed to find genetic variants influencing liver fat content.

Methods Data was acquired from UK Biobank (application 9914). Liver phenotypes were calculated from MRI data by trained analysts using LiverMultiScanTM. We used GEMMA to perform a genome-wide association study (GWAS) of MRI scan measures of liver fat using 8,289 individuals of European ancestry from UK Biobank. We adjusted our analysis for age, sex, BMI, genotyping array, and population structure.

Results We identified two loci in/near PNPLA3 (rs738409, p $\left.=2.1 \times 10^{-41}\right)$ and TM6SF2 (rs58542926, $\mathrm{p}=4.3 \times 10^{-40}$ ) that reached genome-wide significance. We further identified four suggestive loci previously associated with circulating lipid levels, type 2 diabetes and obesity (APOE, GPAM, TRIB1, GCKR). Phenome-wide association analysis (PheWAS) of rs58542926 in TM6SF2 demonstrated positive associations with diabetes, rosacea and liver cirrhosis, and inverse associations with cholesterol, peripheral vascular disease, gout, pulmonary embolism and gallstones. Phenome-wide association analysis of rs738409 in PNPLA3 demonstrated positive associations with liver disease, type 2 diabetes and hypertension, and inverse associations with height, hip circumference, leg and arm fat free mass, cholesterol, and ischaemic heart diseases.

Conclusion This is the first GWAS using MRI determined liver fat content to date. Mechanisms underlying elevated liver fat content contribute to hypertension and type 2 diabetes risk, but may also confer health benefits. The identification of loci previously associated with non-alcoholic fatty liver disease provide genetic validation of the utility of MRI for a fast and non-invasive assessment of liver fat content.
Abstract ATU-08 Table 1 Genome wide $\left(\mathrm{P}<5 \times 10^{-8}\right)$ susceptibility loci for MRI liver fat content.

\begin{tabular}{lllllll}
\hline SNP & Gene & Chr & $\begin{array}{c}\text { Effect } \\
\text { allele }\end{array}$ & EAF & BETA & P \\
\hline rs738409 & [PNPLA3] & 22 & G & 0.26 & 1.153 & $\begin{array}{l}2.1 \mathrm{x} \\
10^{-41}\end{array}$ \\
r558542926 & [TM6SF2] & 19 & T & 0.06 & 1.754 & $4.3 \mathrm{x}$ \\
& & & & & & $10^{-40}$ \\
\hline
\end{tabular}

\section{REFERENCES}

1. Younossi, $Z$ et al. Global burden of NAFLD and NASH: trends, predictions, risk factors and prevention. Nat. Rev. Gastroenterol. Hepatol. 15, 11-20 (2018).

2. Adams, L A, Anstee, Q M, Tilg, H. \& Targher, G. Non-alcoholic fatty liver disease and its relationship with cardiovascular disease and other extrahepatic diseases. Gut (2017). doi:10.1136/gutjnl-2017-313884

\section{ATU-09 OBETICHOLIC ACID TREATMENT IS ASSOCIATED WITH IMPROVED COLLAGEN MORPHOMETRY IN PATIENTS WITH PRIMARY BILIARY CHOLANGITIS}

${ }^{1}$ Andreas E Kremer, ${ }^{2}$ Christopher L Bowlus, ${ }^{3}$ Pierre Bedossa, ${ }^{4}$ Albert Parés, ${ }^{5}$ Lisa M Forman, ${ }^{6}$ Joost PH Drenth, ${ }^{7}$ Stephen Ryder* ${ }^{8}$ Luigi Terracciano, ${ }^{9}$ Yuying Jin, ${ }^{9}$ Alexander Liberman, ${ }^{9}$ Richard Pencek, ${ }^{9}$ Leigh MacConell, ${ }^{10}$ Paul J Pockros. ${ }^{1}$ Department of Medicine I, FriedrichAlexander-University Erlangen-Nürnberg, Erlangen, Germany; ${ }^{2}$ University of California Davis, Sacramento, USA; ${ }^{3}$ Department of Pathology, Physiology and Imaging, University Paris Diderot, Paris, France; ${ }^{4}$ Hospital Clinic, University of Barcelona, CIBERehd, IDIBAPS, Barcelona, Spain; ${ }^{5}$ Division of Gastroenterology-Hepatology, University of Colorado, Aurora, USA; ${ }^{6}$ Department of Gastroenterology and Hepatology, Radboud University Medical Center, Nijmegen, The Netherlands; 'NIHR Nottingham Biomedical Research Centre at Nottingham University Hospitals NHS Trust, Queen's Medical Centre, Nottingham, UKi ${ }^{8}$ Department of Pathology, University of Basel, Basel, Switzerland; 9 Intercept Pharmaceuticals Inc., San Diego, USA; ${ }^{10}$ Division of Gastroenterology/Hepatology, Scripps Clinic and Scripps Translational Science Institute, La Jolla, USA

\subsection{6/gutjnl-2019-BSGAbstracts.209}

Introduction Obeticholic acid (OCA) is a potent, selective FXR agonist approved for the treatment of PBC based upon biochemical improvements predicted to improve transplant-free survival. An optional liver biopsy substudy was conducted in a subset of patients from the Phase 3 POISE trial to determine the impact of OCA treatment on progression of liver fibrosis. Results, using standard histologic grading, showed that the majority of patients treated with OCA either improved or had no worsening of fibrosis over 3 years. New technologies, such as second harmonic generation (SHG) microscopy, allow
A

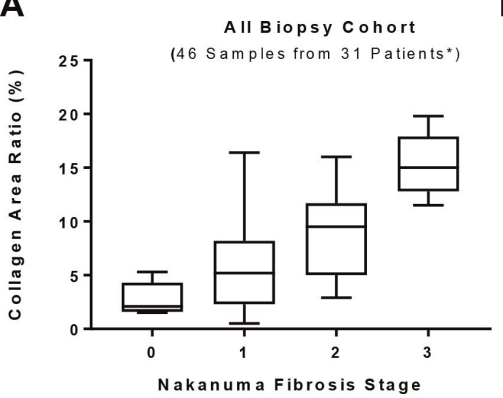

B

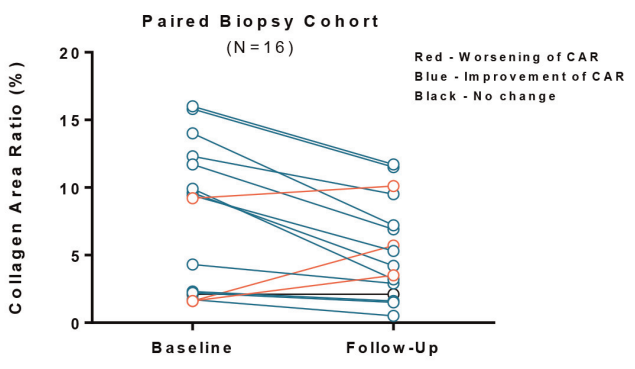

Abstract ATU-09 Figure 1 